tion by ligature or stenosis of the branches of the renal artery. The operation is harmless and very simple. The results obtained by Dr. Janeway show that it is efficient.

\title{
56 (394)
}

A modification of the Riva-Rocci method of determining bloodpressure for use on the dog.

By THEODORE C. JANEWAY.

[From the Rockefeller Institute for Medical Research.]

Previous studies of the blood-pressure changes in living animals, by repeated direct measurements from the femoral or carotid, while accurate from the standpoint of the blood-pressure at the moment of observation, have been of very limited value. When used as a means of following the changes occurring over long periods of time, as in the study of experimental kidney insufficiency, it is questionable whether a single pre-operative reading, with several post-operative ones, afford in themselves any basis for the conclusions drawn. The figures given by Volkmann for the blood-pressure of different animal species show readings from the dog of $104,123,143,157,166$ and $172 \mathrm{~mm}$., a variation so wide that, in the light of our knowledge of the fluctuations of blood-pressure in man, it suggests strongly the fallacy of any conclusions drawn from a comparison of two or three measurements at long intervals.

To obtain some more definite idea of the changes occurring from day to day in experimental animals, I have endeavored to apply to the dog the commonly employed clinical methods. After various attempts, the most satisfactory method was found to be a modified Riva-Rocci cuff applied to the lower foreleg, the pulse being palpated in the artery at the bend of the ankle or in the plantar aspect of the paw. A rubber bag $7.5 \times 15 \mathrm{~cm}$., with a slightly larger outer leather cuff, will fit almost any dog, the foreleg being not less than $8 \mathrm{~cm}$. in length, and from II to $14 \mathrm{~cm}$. in circumference in a large number of laboratory dogs examined. For small dogs a cuff $5 \times$ I I cm. is adequate. Measurements are greatly facilitated by using a pressure bottle connected with the cuff and the manometer through valves operated by foot pedals, 
leaving both hands free, in place of the usual rubber bulb or Politzer bag as the source of pressure.

The greatest difficulty is the satisfactory palpation of the small pulse in the foot. In certain dogs it is impossible, and animals must be selected that possess a reasonably large and superficial artery. It is impossible to appreciate the return of the pulse after obliteration, but with practice, if the animal can be kept quiet, the obliteration of the pulse can be appreciated within perhaps 10 or I $5 \mathrm{~mm}$. limits of error, always on the side of under-estimation. When the foot is cold, it should be wrapped in warm cloths to dilate the vessels, before taking readings.

A number of experiments, in which I have followed the pressure changes during an operation coincidently with a direct carotid tracing, show that one can follow fairly rapid and marked fluctuations of blood-pressure in this way, with reasonable certainty. The results have no absolute, but, I am convinced, a real relative value. For the solution of such problems as the one studied by Pässler and Heineke, and which Carrel and I are engaged in, I believe that frequent approximate blood-pressure observations are of more significance than a few isolated, though accurate, measurements.

\section{$57(395)$}

Note on the blood-pressure changes following reduction of the renal arterial circulation.

\section{By THEODORE C. JANEWAY.}

\section{[From the Rockefeller Institute for Medical Research.]}

Of the various workers who have studied the effects of reduction of kidney substance, only Pässler and Heineke record systematic blood-pressure observations. They were able to make direct measurements in the femoral on five dogs before and after operation, and reported a rise in pressure in all, the smallest increase being I $5 \mathrm{~mm}$., the greatest $29 \mathrm{~mm}$., and the average $21.5 \mathrm{~mm}$. These figures are based on the comparison of single readings before operation with one or more after operation, and are open to the objections I have previously urged. Because of the small number of reported observations in this field, I hope to be pardoned for presenting my still very incomplete studies at this time, in order 